\title{
Multiple Sensors, Voting Methods and Target Value Analysis
}

\author{
J.R. Parker \\ Laboratory for Computer Vision \\ Department of Computer Science \\ University of Calgary \\ Calgary, Alberta, CANADA
}

\begin{abstract}
Voting techniques for high level sensor/data fusion are explored here, with examples from character recognition and target value analysis. High degrees of reliability can be achieved, and the method can be applied to various kinds of data. The only requirement is that a ranking of the targets be extracted from the sensors.
\end{abstract}

Keywords: decision fusion, voting techniques, value analysis

\subsection{Introduction}

There are many levels of abstraction at which sensor fusion can be applied. Multiple sensors, whether identical or responsive to various stimuli, can be integrated in many different ways, and multi-sensor fusion could refer to any stage in any of the possible integration processes. At low levels, individual measurements (pixels, if the sensor data is in image form) can be combined. Feature level and object level fusion require more sensor specific processing of the data, and can more effectively be used for tracking shapes over time. At still higher levels of abstraction we can combine information from quite different sensors at various degrees of organization, possibly using a blackboard system or knowledge base to provide context for the fusion.

In all instances above the goal is to assess information from a variety of input sources having various properties into a simple coherent result. Always the best answer is required, sometimes in the face of contradictory data. One technique that can be used in many cases is that of voting.

Simple voting techniques have been discussed before, for various purposes all relating to the combining of independently computed results[1,6, 7,11]. These are quick, but have serious limitations, not the least of which is that they tend to discard much of the information presented to them. For example, consider the following problem, similar to that in [1]: small raster images corresponding to hand printed digits (glyphs) are available, and it is desired to classify those as being a ' 1 ', a ' 2 ' and so on for each case. We have five different methods at our disposal, having the following recognition rates on a sample of 1000 glyphs:

\section{Table 1: Success rates for character} recognition methods

\begin{tabular}{|l|l|}
\hline Method & $\begin{array}{l}\text { Recognition } \\
\text { Rate }\end{array}$ \\
\hline 1 & $94.2 \%$ \\
\hline 2 & $93.9 \%$ \\
\hline 3 & $94.3 \%$ \\
\hline 4 & $95.4 \%$ \\
\hline 5 & $94.9 \%$ \\
\hline
\end{tabular}

Since there are five different estimates of the result for each sample image we could choose the best method an use it only, or an effort could be made to combine the individual classifications. For example, a simple majority vote could be used; if at least three out of the five classifiers agree on the classification, the 
majority classification is used as the final result. If the combined result is not better than that obtained by any of the individual classifiers then we have wasted our effort. Indeed not, since the result of the majority vote in the case described above turned out to be a recognition rate of $99.6 \%$. This situation is real, and is discussed in detail in [8].

The majority vote discards information in the sense that, first, dissenting votes that agree should have more weight than those that disagree, but do not. Also, in most cases, a classifier could rank the likely classifications in order of likelihood (EG Probably a '2', but could be a ' 7 ', and is less likely a ' 1 '). Also, in the example, the 'sensors' are really methods for character recognition, and all have exactly the same goal. A sensor fusion problem frequently uses sensors that measure completely different things. In the following two sections both of these issues will be dealt with: use of ranked data in voting, and use of diverse data.

\section{0 Voting For Fusion Of Ranked Data}

For the purposes of discussion, sensor data can be placed into one of three classes. Type I data is simply a single statement such as 'the glyph is a digit 2', or 'the object is an F18 aircraft'. Type II data is a list of possibilities in descending order of likelihood: 'the object is most likely an F15, but could be an F14, less likely an F18, and less likely still to be an F104.' Type III data assigns actual probabilities to the targets, and this is rarely possible. If sufficient information is available, each type of data can be converted into each other type. For example, converting a single response to a rank cannot be done in a completely general and reliable fashion, but an approximation can be had based on the measured past performance of the particular algorithm using the confusion matrix. Converting type II to type I data is done simply by choosing the highest ranked element in each set.

The problem encountered when attempting to merge ranked responses is as follows: given $\mathrm{M}$ rankings, each having $\mathrm{N}$ choices, which choice has the largest degree of support?
For example, consider the following 3 voter/4 choice problem [10]:

Voter 1: a b c d

Voter 2: $\mathrm{c} \mathrm{a} \mathrm{b} \mathrm{d}$

Voter 3: b d c a

This case has no majority winner; $a, b$ and $\mathrm{c}$ each get one first place vote. Intuitively, it seems reasonable to use the second place votes in this case to see if the situation resolves itself. In this case $b$ receives two second place votes to a's one, which would tend to support $b$ as the overall choice. In the general case there are a number of techniques for merging rank-ordered votes, four of which will be discussed here.

The Borda count [3] is an ancient scheme for resolving this kind of situation. Each alternative is given a number of points depending on where in the ranking it has been placed. A selection is given no points for placing last, one point for placing next to last, and so on, up to N-1 points for placing first. In other words, the number of points given to a selection is the number of classes below it in the ranking. However, the Borda count does have a problem that might be considered serious. Consider the following 5 voter 3 choice problem:

Voter $1: \mathrm{a} \mathrm{b} \mathrm{c}$

Voter 2:a b c

Voter 3:a b c

Voter 4:b c a

Voter 5:b c a

The Borda counts are $a=6, b=7, c=2$, which selects $b$ as the winner. However, a simple majority of the first place votes would have selected a! This violates the so-called majority criterion [10]:

If a majority of voters have an alternative $X$ as their first choice, a voting rule should choose X.

This is a weaker version of the Condorcet Winner Criterion [4]:

If there is an alternative $X$ which could obtain a majority of votes in pair-wise contests against every other alternative, a voting rule should choose $X$ as the winner.

The Condorcet criterion can be used alone as a way to select a result from 
ranked data, but it tends to produce a large number of tie votes.

One way to deal with ties, and to ensure a majority in simple voting methods, is an elimination process, common in political conventions. Votes are carried out multiple times, each time removing the least popular selection. While easily understood, this violates the Condorcet criterion and also fails the monotonicity criterion:

If $X$ is a winner under a voting rule, and one or more voters change their votes in a way favorable to $X$ without changing the order in which they prefer any other alternative, then $X$ should still be the winner. This should so obviously be true that no rule violating this principle will be considered.

With the monotonicity criterion in mind, two strategies become interesting. The Black[2] strategy chooses the winner by the Condorcet criterion if such a winner exists; if not, the Borda winner is chosen. This is appealing in its simplicity, and can be shown to be monotonic. The Copeland strategy computes, for each option, the number of pair-wise wins of that option with all other options (call this $\mathrm{N}_{\mathrm{W}}$ ) and the number of pair-wise losses $\left(\mathrm{N}_{1}\right)$. The winner is the option for which $\mathrm{N}_{\mathrm{w}}-\mathrm{N}_{1}$ is a maximum.

The results or these voting schemes (Borda, Black, and Copeland), as applied to the character recognition problem discussed previously, are summarized in Table 2.

\section{Table 2: Results of the Voting Rules for Rank Ordering}

\begin{tabular}{|l|l|}
\hline Rule & Recognition \\
\hline Borda & $99.9 \%$ \\
\hline Black & $99.9 \%$ \\
\hline Copeland & $99.6 \%$ \\
\hline
\end{tabular}

From this table it would appear that the Borda scheme is tied with Black's, and then Copeland's. It is important to recall that Borda does not satisfy the majority criterion. If the results from the classifier are simple single-valued class specifications, then the overall classification from the multiple classifier can be obtained by a simple majority vote. Finally, in cases where actual probabilities are produced by a classifier, the overall result can be given by the class having the largest average value of all of the classes.

\subsection{A Target Value Analysis/ Threat Assessment Problem}

An attempt will now be made to evaluate the voting methods discussed so far as applied to a simple tactical problem. Consider the sensor array on a fighter aircraft; there is a large collection of potential sensors, but an easy to understand set is:

Infrared sensor, which returns a number between 0 and 1 , the degree to which IR signature agrees with that of hostile target.

Optical sensor, which looks at the size and shape of each target and, again, returns a numbers between 0 and 1 , the degree to which the signature matches a hostile one.

Doppler radar, which returns both the speed of approach of and range to the target.

Radio - friend or foe.

The simulation of a test scenario contains ten targets, the sensor data for which appears in Table 3 below, and a diagram of which appears as Figure 1. For analysis, range values are inverted; this means that the largest value indicates the greatest threat in all cases.

\section{Table 3: Sensor Data For Threat Analysis Problem}

\begin{tabular}{|l|l|l|l|l|l|}
\hline Target & $\begin{array}{l}\text { IR } \\
(\mathbf{0}-\mathbf{1})\end{array}$ & $\begin{array}{l}\text { Optical } \\
(\mathbf{0 - 1})\end{array}$ & $\begin{array}{l}\text { Velocity } \\
(\mathbf{M} / \mathbf{s})\end{array}$ & $\begin{array}{l}\text { Range } \\
(\mathbf{M})\end{array}$ & $\begin{array}{l}\mathbf{F} / \mathbf{F} \\
\mathbf{( 1 / 0 )}\end{array}$ \\
\hline $\mathrm{t} 1$ & 0.65 & 0.2 & 120 & 3500 & 0 \\
\hline $\mathrm{t} 2$ & 0.53 & 0.4 & 79 & 1200 & 1 \\
\hline $\mathrm{t} 3$ & 0.60 & - & -35 & 2250 & 0 \\
\hline $\mathrm{t} 4$ & 0.81 & - & -93 & 4600 & 0 \\
\hline $\mathrm{t} 5$ & 0.40 & 0.31 & 0.5 & 101 & 1 \\
\hline $\mathrm{t} 6$ & 0.80 & 0.51 & -21 & 1032 & 0 \\
\hline $\mathrm{t} 7$ & 0.21 & 0.56 & 114 & 2100 & 0 \\
\hline $\mathrm{t} 8$ & 0.75 & - & 92 & 5162 & 0 \\
\hline $\mathrm{t} 9$ & 0.41 & 0.35 & -.26 & 210 & 1 \\
\hline $\mathrm{t} 10$ & 0.68 & 0.52 & 97 & 2261 & 0 \\
\hline
\end{tabular}


Voting methods will operate on columns of the Table 3, which will be called election vectors. The first four data columns are used for voting, with the last used to eliminate friendly craft from consideration. A simple majority vote, using only the maximum value of each sensor, yields no result - the largest value of in each column occurs with a different target.

Ranking the sensor values for this problem means simply sorting them into descending order, keeping track of the corresponding target in each case. The ranked values are given, in turn, to the Borda, Condorcet, Black, and Copeland methods, which were implemented in the $C$ language on a small 486/PC.

The Borda ranking for this problem, with counts in parentheses, is:

$\begin{array}{ll}1 & \mathrm{~T}_{6}(24), \mathrm{T}_{10}(24), \\ 2 & \mathrm{~T}_{7}(23) \\ 3 & \mathrm{~T}_{1}(20) \\ 4 & \mathrm{~T}_{8}(15) \\ 5 & \mathrm{~T}_{4}(12) \\ 6 & \mathrm{~T}_{3}(9) \\ \text { friendly } & {\left[\mathrm{T}_{2}, \mathrm{~T}_{5}, \mathrm{~T}_{9}\right]}\end{array}$

The Condorcet ranking for the problem differs only slightly:

$\begin{array}{ll}1 & \mathrm{~T}_{10} \\ 2 & \mathrm{~T}_{6}\end{array}$

Figure 1 - A graphical (Radar) display of the simulated target data used to test the voting schemes. We (our aircraft) is unlabeled, at the origin of the drawn axes.

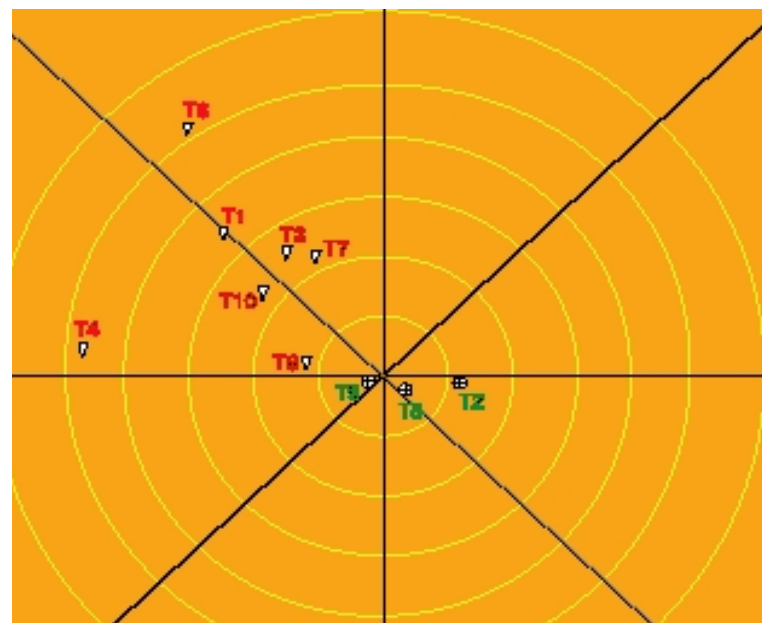

$$
\begin{array}{ll}
3 & \mathrm{~T}_{7} \\
4 & \mathrm{~T}_{1} \\
5 & \left(\mathrm{~T}_{8}=\mathrm{T}_{4}=\mathrm{T}_{3}\right) \\
\text { friendly } & {\left[\mathrm{T}_{2}, \mathrm{~T}_{9}, \mathrm{~T}_{5}\right]}
\end{array}
$$

The difference is really nothing, since the first two places in the Borda ranking are tied; the ties in the Condorcet ranking simply occur at a different place. The Copeland ranking is:

$\begin{array}{ll}1 & \left(\mathrm{~T}_{6}=\mathrm{T}_{7}\right) \\ 2 & \mathrm{~T}_{10} \\ 3 & \mathrm{~T}_{1} \\ 4 & \left(\mathrm{~T}_{3}=\mathrm{T}_{4}=\mathrm{T}_{8}\right) \\ \text { friendly } & {\left[\mathrm{T}_{2}, \mathrm{~T}_{9}, \mathrm{~T}_{5}\right]}\end{array}$

This is fairly consistent with the previous rankings, the difference being the position of target 7. Finally, the Black ranking of this data set is:

$\begin{array}{ll}1 & \mathrm{~T}_{6} \\ 2 & \mathrm{~T}_{10} \\ 3 & \mathrm{~T}_{1} \\ 4 & \mathrm{~T}_{7} \\ 5 & \left(\mathrm{~T}_{8}=\mathrm{T}_{4}=\mathrm{T}_{3}\right) \\ \text { friendly } & {\left[\mathrm{T}_{2}, \mathrm{~T}_{9}, \mathrm{~T}_{5}\right]}\end{array}$

Based on simulations of the methods with known data, the most reliable scheme would be selected for use with the sensors in question. Past experience in the context of character recognition suggests the use of the Black scheme, but in any case there is a general agreement. A meta-vote could also be done, applying a Borda count or majority vote to the new ranking from the voting methods. This would clearly rank the most threatening targets as $\mathrm{T}_{\mathbf{6}}, \mathrm{T}_{\mathbf{1 0}}, \mathrm{T}_{7}$.

\subsection{Conclusions}

The voting schemes described here would all be useful in many situations for highlevel senor/data fusion, and any would be superior to a simple majority vote, which simply does not work for the target data provided. Fault tolerance is maintained, as any non-functional sensor would not contribute to the rankings, and the result of the vote would be the best available under the circumstances. Note 
that the test data is missing some optical information for distant targets and the voting still arrives at a reasonable conclusion.

More work should be done on the mathematical validity of a meta-vote, and on the behavior of the threat assessment as a function of time. For example, what is the meaning of a target with a large gradient?

\subsection{References}

[1] Baraghimian, G.A., Klinger, A., Preference Voting for Sensor Fusion, SPIE Sensor Fusion III, Orlando, Fl. April 19-20, 1990.

[2] Black, D., The Theory of Committees and Elections, Cambridge University Press, 1958.

[3] Borda, Jean-Charles de., Memoire sur les Elections au Scrutin, Histoire de l'Academie Royale des Sciences, Paris, 1781.

[4] Condorcet, Marquis de., Essai sur l'application de l'analyse a la probabilite des decisions rendues a la pluralite des voix, Paris, 1785.

[5] Kimura, F. and Shridhar, M., Handwritten Numeral Recognition Based On Multiple Algorithms, Pattern Recognition, Vol. 24 No 10, 1991.

[6] Parker, J.R., Practical Computer Vision Using C, John Wiley \& Sons, N.Y., 1994.

[7] Parker, J.R., Vector Templates and Handprinted Character Recognition, Proc. 12th IAPR Conference on Pattern Recognition, Jerusalem, Israel. Oct 9-13, 1994.

[8] Parker, J.R., Recognition of Hand Printed Digits Using Multiple/Parallel Methods, Third Golden West International Conference on Intelligent Systems, Las Vegas, June 6$9 / 94$.

[9] Parker, J.R., Voting Methods for Multiple Autonomous Agents, Perth, Australia, 1996.

[10] Straffin, P.D. Jr., Topics in the Theory of Voting, Birkhauser, Boston, 1980.

[11] Xu, L., Krzyzak, A., and Suen, C.Y., Methods Of Combining Multiple Classifiers And Their Application To Handwriting
Recognition, IEEE Transactions on Systems, Man, and Cybernetics, Vol. 22 No. 3, May/June 1992. 